\title{
Frizzled Gene Expression and Negative Regulation of Canonical WNT- $\beta \beta$-catenin Signaling in Mouse F9 Teratocarcinoma Cells
}

\begin{tabular}{|r|l|}
\hline Journal: & Biochemistry and Cell Biology \\
\hline Manuscript ID & bcb-2016-0150 \\
\hline Manuscript Type: & Article \\
\hline Complete List of Authors: & $\begin{array}{l}\text { Golenia, Gregory; University of Western Ontario, Biology, Molecular } \\
\text { Genetics Unit } \\
\text { Gatie, Mohamed; University of Western Ontario, Biology, Molecular } \\
\text { Genetics Unit } \\
\text { Kelly, Gregory; University of Western Ontario, Biology, Molecular Genetics } \\
\text { Unit; Child Health Research Institute; Ontario Institute for Regenerative } \\
\text { Medicine }\end{array}$ \\
\hline Keyword: & WNT, $\beta$-catenin, Mouse Embryo, Extraembryonic Endoderm, Dickkopf \\
\hline &
\end{tabular}

\section{SCHOLARONE" \\ Manuscripts}




\section{Frizzled Gene Expression and Negative Regulation of Canonical WNT- $\beta$-catenin Signaling in Mouse F9 Teratocarcinoma Cells}

Gregory Golenia $^{1}$, Mohamed I. Gatie ${ }^{1}$ and Gregory M. Kelly $y^{1,2,3^{*}}$

1 Department of Biology, Molecular Genetics Unit, Western University, London, ON, Canada N6A 5B7. 2. Child Health Research Institute, London, ON N6C 2V5, and 3. Ontario Institute for Regenerative Medicine, Toronto, Ontario, Canada M5G 0A4.

Email: gkelly@uwo.ca

Keywords: WNT, ß-catenin, Mouse Embryo, Extraembryonic Endoderm, Dickkopf 
Abstract: Mouse F9 cells differentiate into primitive endoderm (PrE) following the activation of the canonical WNT/ $\beta$-catenin pathway. The upregulation of Wnt6 and activation of $\beta$-cateninTCF/LEF-dependent transcription is known to accompany differentiation, but the Frizzled (FZD) receptor responsible for transducing the WNT6 signal is not known. Eight of the ten Fzd genes were found to be expressed in F9 cells, with $\mathrm{Fzd} 7$ being the most highly expressed, and chosen for further analysis. To alter steady-state $F z d 7$ levels and test the effect this has on differentiation, siRNA and overexpression approaches were used to knockdown and ectopically express the $F z d 7$ message, respectively. siRNA knock down of $F z d 7$ resulted in reduced DAB2 levels, and the overexpression activated a TCF-LEF reporter, but neither approach affected differentiation. Our focus turned to how canonical WNT6 signaling was attenuated to allow PrE cells to form PE. Dkk1, encoding a WNT antagonist, was examined and results showed that its expression increased in F9 cells treated with RA or overexpressing Wnt6. F9 cells overexpressing human $D K K 1$ or treated with DKK1-conditioned medium and then treated with RA failed to differentiate, indicating that a negative feedback loop involving WNT6 and DKK1 attenuates canonical WNT/ $\beta$-catenin signaling, thereby allowing PE cells to differentiate. 


\section{Introduction}

During mouse early embryogenesis the blastocyst consists of the trophectoderm, the epiblast, and the primitive endoderm (PrE) (Rossant and Tam 2009). PrE differentiates into parietal endoderm (PE) and visceral endoderm (VE), which develop into the parietal and visceral yolk sacs, respectively (Boiani and Scholer 2005). PE formation occurs around implantation, and although difficult to study in vivo, there are alternative in vitro models including the F9 teratocarcinoma cell line (Strickland et al. 1980). F9 cells treated with retinoic acid (RA) undergo changes that mimic the formation of PrE (Strickland and Mahdavi 1978; Yang et al. 2011). Subsequent addition of db-cAMP induces PE, and if grown in suspension culture, $\operatorname{PrE}$ cells differentiate into VE (LaMonica et al. 2009; Verheijen et al. 1999). One of several pathways activated by RA involves canonical WNT/ $\beta$-catenin signaling (Krawetz and Kelly 2008; Liu et al. 2002).

WNTs are secreted glycoproteins that elicit numerous cellular responses in vertebrate and invertebrate animals. The nineteen mammalian WNTs that bind to one or more of the ten plasma membrane spanning Frizzled (FZD) receptors signal through the canonical WNT/ $\beta$-catenin, and/or the non-canonical WNT/Ca ${ }^{2+}$ or planar cell polarity (PCP) pathways (Logan and Nusse 2004; Moon 2005). The effects of pathway activation are pleiotropic and include stimulation of cell proliferation, cell motility and control of cell fate and differentiation (Logan and Nusse 2004). In F9 cells, RA treatment up-regulates Wnt6, and the subsequent activation of the canonical pathway allows for the interaction of $\beta$-catenin with the T-cell-factor-lymphoid enhancer factor (TCF-LEF) family of transcription factors. This interaction promotes the expression of genes necessary for PrE induction (Krawetz and Kelly 2008), and as found here, genes involved in attenuating the WNT6 signal.

F9 cells require the activation of canonical WNT signaling to induce cells to form PrE, and non-canonical WNT signaling pathways to complete differentiation (Krawetz and Kelly 2008; LaMonica et al. 2009). Therefore, either a WNT and/or a receptor that has the potential to activate both of these WNT signaling pathways would be ideally suited to induce the differentiation of F9 cells into PE. Both FZD7 and WNT6 mediate canonical and non-canonical signaling in several cell types, and there is a link between the two in embryos (Beaton et al. 2016; King et al. 2012; Linker et al. 2005; Schmidt et al. 2007; Zhang et al. 2013). Based on this 
evidence, we postulated that FZD7 was the WNT6 receptor that transduces the signal to induce F9 cells to differentiate towards PrE.

In addition to WNT6 acting through a FZD receptor to regulate genes required for the differentiation to PrE, mechanisms are in place to attenuate the canonical WNT signal for these cells to differentiate into PE and VE (LaMonica et al. 2009; Schlupf and Steinbeisser 2014; Shibamoto et al. 2004). There is evidence that F9 cells express intracellular and extracellular inhibitors of WNT signaling during differentiation (Shibamoto et al. 2004; Zhuang et al. 2003). Dickkopf (DKK) proteins are extracellular antagonists of WNT signaling (Kawano and Kypta 2003), and the expression of Dkk1 in F9 cells was reported to be RA-induced in VE cells (Shibamoto et al. 2004).

Our studies have shown that eight of ten $F z d$ genes were expressed in F9 cells and $F z d 7$ is the most highly expressed. Genetic manipulations of $F z d 7$ were not sufficient to affect the induction of F9 cells into PrE. Nevertheless, we have identified a negative feedback loop initiated by canonical WNT6 signaling involving the WNT antagonist, DKK1, that permit cells to continue their differentiation from PrE to PE.

\section{Materials and methods}

\section{Cell culture, reverse transfection, and chemical treatments}

Mouse F9 teratocarcinoma cells (ATCC) were cultured in Dulbecco's Modified Eagle Medium (DMEM) (Gibco) supplemented with 1\% penicillin-streptomycin (P/S) (Gibco) and $10 \%$ fetal bovine serum (FBS) (Gibco) and incubated at $37^{\circ} \mathrm{C}$ and $5 \% \mathrm{CO}_{2}$. Cells were cultured in $35 \mathrm{~mm}$ plates for transfection or $60 \mathrm{~mm}$ plates (Nunc Thermoscientific or Sarstedt) for protein and/or RNA isolation. Cells were transfected with empty vector, $p c D N A 3.1-F z d 7$ (B. Cadesky, Western University), pcDNA3.1-Wnt6, pcDNA3.1-Myc-DKK1 (Dr. Y. Li, Southern Research Institute, Birmingham, AL), $p B A R L$ (Dr. S. Angers, University of Toronto) and $p R L-T K$ (Dr. R. Dekoter, Western University), using Lipofectamine 2000 (Life Technologies).

For the $F z d 7$ knockdown, three 27-mer antisense RNA sequences (Origene) were pooled together at $10 \mathrm{nM}$ each. Approximately $2.5 \times 10^{5}$ cells were added to $2 \mathrm{~mL}$ DMEM with serum and antibiotics and seeded directly into $35 \mathrm{~mm}$ dishes containing the Opti-MEM transfection mix. Medium was replaced after $6 \mathrm{~h}$ and approximately $1 \times 10^{5}$ cells were plated into $60 \mathrm{~mm}$ dishes after $24 \mathrm{~h}$ and selected for antibiotic resistance with $1.75 \mathrm{mg} / \mathrm{mL}$ G418 sulfate (Sigma). 
Cells transfected with $p c D N A 3.1-M y c-D K K 1$ or treated with MYC-DKK1-conditioned medium were treated with DMSO or $5 \times 10^{-8} \mathrm{M}$ RA for 4 days. For all other experiments, cells were treated with DMSO or $10^{-7} \mathrm{M}$ RA for 3 or 4 days.

\section{Reverse-transcription PCR and quantitative reverse-transcription PCR}

Total RNA was isolated using the RNeasy Mini Kit (Qiagen) and used as a template for first-strand cDNA synthesis, using the High Capacity cDNA Reverse Transcription kit (Applied Biosystems). RNA was isolated following treatment of F9 cells with DMSO, RA (1, 2, 3, and 4 days post-treatment) or RA and db-cAMP (1mM), and for RT-PCR and qRT-PCR with Fzd primers, DNase digestion was performed using the RNase-Free DNase kit (Qiagen). Purity and concentration of RNA was analyzed using a NanoDrop2000C Spectrophotometer (Thermo Scientific). PCR reactions were analyzed on 1\% agarose gels containing RedSafe Nucleic Acid Stain (iNtRON Biotechnology) and amplicons were visualized using a FluorChem 8900 Gel Doc System (Alpha Innotech). All reactions were performed using the following thermo-cycling conditions for $35-40$ cycles: $94^{\circ} \mathrm{C}$ for $30 \mathrm{~s}$, the primer-specific annealing temperature for $30 \mathrm{~s}$ and $72^{\circ} \mathrm{C}$ extension for 30s. All amplicons were sequenced (Robarts Research Institute, Western University) and aligned to annotated sequences using the NCBI Basic Local Alignment Search Tool (BLAST) to confirm their identity. Primers sequences and annealing temperatures are listed in the Supplemental Information.

For qRT-PCR analysis, total RNA was isolated and reverse-transcribed into first-strand cDNA as described above. For $F z d$ expression analysis, qRT-PCR was performed using the Rotor-Gene 3000 (Corbett Research) and Power SYBR Green Master Mix (Applied Biosystems). For all other qRT-PCR reactions, the CFX Connect Real-Time PCR Detection System (Bio-Rad) was used with the SensiFAST SYBR No-ROX Kit (Bioline). Each qRT-PCR reaction contained $10 \mu \mathrm{L}$ of Power SYBR Green Master Mix or SensiFAST SYBR, 500nM of each primer, and $1 \mu \mathrm{L}$ of cDNA template. Amplification plots were analyzed using the RotorGene or CFX Manager software. Gene expression was quantified using the comparative cycle threshold $(\mathrm{Ct})$ method and displayed as gene expression relative to the constitutively active $L 14$ gene $\left(2^{-\Delta \mathrm{Ct}}\right)$ or as fold-change $\left(2^{-\Delta \Delta \mathrm{Ct}}\right)$ relative to DMSO or empty vector control. 


\section{Immunoblot analysis}

Protein lysates were prepared in RIPA buffer containing 150mM sodium chloride, $1 \%$ NP-40, 0.5\% sodium deoxycholate, $0.1 \%$ SDS, 50mM Tris- $\mathrm{HCl}$ and $1 \mathrm{x}$ Halt Protease inhibitor cocktail (Thermo Scientific). Protein concentrations were quantified using the Detergent Compatible protein assay (Bio-Rad) and Modulus II Microplate Multimode Reader (Turner Biosystems). Approximately $20 \mu \mathrm{g}$ of protein were separated on $10 \%$ denaturing polyacrylamide gels for $2.5 \mathrm{~h}$ at $100 \mathrm{~V}$. Following electrophoresis, the proteins were electrophoretically transferred to polyvinylidene fluoride membranes (Pall Corporation) overnight at $4^{\circ} \mathrm{C}$ and $20 \mathrm{~V}$ in Tris-glycine transfer buffer containing 20\% methanol. The membranes were blocked in Trisbuffered saline containing $0.1 \%$ Tween 20 (TBS-T) and 5\% skim milk for $1 \mathrm{~h}$ at room temperature. Membranes were probed overnight at $4{ }^{\circ} \mathrm{C}$ with the following primary antibodies diluted in 5\% bovine serum albumin (BSA) containing TBS-T: TROMA-1 $(1: 5 ; 55 \mathrm{kDa}$, Developmental Studies Hybridoma Bank), DAB2 (1:10000; 96kDa, BD Transduction Laboratories), $\beta$-actin (1:5000; 47kDa, Santa Cruz) and c-MYC (9E10) (1:50, approximately 39 $\mathrm{kDa}$, Santa Cruz). Membranes were washed in TBS-T (three washes for five minutes each) and then probed with either HRP-conjugated goat anti-rat or anti-mouse secondary antibody in 5\% skim milk in TBS-T for $2 \mathrm{~h}$ at room temperature. Following three additional five minute washes with TBS-T, signals were detected using the Supersignal West Pico Chemiluminescent Detection Kit (Pierce), then captured on a Molecular Imager Gel Doc XR System (Bio-Rad) and imaged with Quantity One Software. Densitometry analysis was performed using ImageJ software.

\section{TCF/LEF reporter assay}

Cells transfected with $p B A R L$ and then treated with DMSO or $10^{-7} \mathrm{M}$ RA, or cotransfected with $p B A R L$ and $p c D N A 3.1-E V$ (empty vector) or $p c D N A 3.1-F z d 7$ in equal amounts, were collected $48 \mathrm{~h}$ post-treatment or post-transfection. Cells were also co-transfected with $p R L$ $T K$ (Renilla luciferase) to normalize luciferase levels. Lysates were collected by shaking cells in $1 \mathrm{X}$ Passive Lysis Buffer (Promega) for 15min at room temperature. Luciferase activity was quantified using the Dual Luciferase Assay Reporter System (Promega) and the Modulus II Multimode Microplate Reader (Promega). Firefly luciferase values were normalized to Renilla luciferase values. 


\section{MYC-DKK1 conditioned medium}

HEK-293 cells were grown to $90 \%$ confluence in $60 \mathrm{~mm}$ culture dishes and maintained in DMEM with $10 \% \mathrm{FBS}$ and $1 \% \mathrm{P} / \mathrm{S}$. Cells were transfected using $10 \mu \mathrm{L}$ Lipofectamine 2000 with $4 \mu \mathrm{g} p c D N A 3.1-E V$ (empty vector) or $p c D N A 3.1-M y c-D K K 1$. Medium was replaced with complete DMEM after $6 \mathrm{~h}$ and replaced again with serum-free medium containing $1 \% \mathrm{P} / \mathrm{S}$ after $24 \mathrm{~h}$. The medium was collected after $48 \mathrm{~h}$ and centrifuged at $10,000 \mathrm{x} \mathrm{g}$ for $10 \mathrm{~min}$ at $4{ }^{\circ} \mathrm{C}$ to pellet cellular debris. The supernatant was filter sterilized using $0.2 \mu \mathrm{m}$ Acrodisc Syringe Filters (Pall Corporation) and concentrated using Amicon Ultra-4 Centrifugal Filter Units (10,000 nominal molecular weight limit-EMD Millipore). Approximately $1.2 \times 10^{4} \mathrm{~F} 9$ cells were seeded in 12-well plates and co-treated with $5 \times 10^{-8} \mathrm{M}$ RA and $10 \mu \mathrm{L}$ of media concentrate from $p c D N A 3.1$ $E V$ or $p c D N A 3.1-M y c-D K K 1$-transfected cells. Cells were supplemented with $10 \mu \mathrm{L}$ of concentrated medium every day for a total of 4 days. For immunoblot analysis of concentrated conditioned medium, 5,10 or $15 \mu \mathrm{L}$ of medium from $p c D N A 3.1-E V$ or $p c D N A 3.1-M y c-D K K 1$ transfected cells were resolved by electrophoresis on $10 \%$ denaturing polyacrylamide gels, transferred to PVDF membranes and probed using the 9E10 antibody (Santa Cruz).

\section{Statistical analysis}

Densitometric analyses of immunoblots, qRT-PCR data, and luciferase assay data were compiled from three independent biological replicates. Comparisons of data between the control and treated, or transfected groups were performed using a one-way ANOVA and Tukey's honest significant difference (HSD) post-hoc test or a Student's t-test (SPSS Statistics for Windows Version 19.0, IBM Corp. Released 2010, Armonk, NY). One-way ANOVA followed by Tukey's HSD test was used for the statistical analysis of the luciferase assay data, Dkk1 and Dab2 qRTPCR data, and densitometry data of the TROMA-1 and DAB2 immunoblots from the Fzd7 knockdown and overexpression experiments. Student's t-test was used for statistical analysis of all other data. P-values were one-sided and considered statistically significant at the 0.05 level. Statistical data are presented as the mean \pm S.E.

\section{Results and Discussion}

WNT signaling pathways, which are evolutionarily conserved in Metazoans, play instrumental roles in a number of events including cell migration, cell fate determination, 
organogenesis, stem cell pluripotency, tissue homeostasis, and when dysregulated, fibrosis and carcinogenesis. The result of a WNT ligand binding to its cognate FZD receptor is the initiation of one or several intracellular signaling cascades, which include the canonical WNT/ $\beta$-catenindependent pathway and the non-canonical, $\beta$-catenin-independent planar cell polarity (PCP) and $\mathrm{WNT} / \mathrm{Ca}^{2+}$ pathways. There are ten different FZD receptors and nineteen WNT ligands that have been identified in mammals, allowing for a remarkable number of receptor-ligand combinations that complicates the understanding of how one particular WNT pathway is activated preferentially over another (Moon 2005). This preferential selectivity of a signaling branch is thought to depend on several factors such as the intrinsic properties of WNTs and FZDs themselves, as well as presence of the receptors and co-receptors in a cell-dependent context (Dijksterhuis et al. 2014).

In this study F9 cells were used to investigate how the canonical WNT/ $\beta$-catenin signaling pathway, and specifically the role of the FZD receptors, specify relatively naïve cells to not only adopt the PrE fate, but how the signal is attenuated to permit these cells to complete their differentiation to PE. F9 cells differentiate into PrE when treated with RA, and this is accompanied by the upregulation of Wnt6 and activation of the canonical $\mathrm{WNT} / \beta$-catenin pathway (Krawetz and Kelly 2008). Wnt6 is upregulated by the transcription factors GATA6 and FOXA2, which bind to and activate the Wnt6 promoter in F9 cells (Hwang and Kelly 2012). Although the involvement for WNT6 in RA induced differentiation is clear, the receptor(s) responsible for binding WNT6 and transducing the differentiation signal, and then for its attenuation is/are unknown.

\section{Fzd expression in F9 and PrE cells}

It was reported previously that all ten members of the $F z d$ family are expressed in F9 cells (Okoye et al. 2008). In light of the fact that this previous study also reported that eighteen of the nineteen Wnt genes were also expressed in F9 cells, despite our ability to only detect Wnt6 (Krawetz and Kelly 2008), it was decided to re-evaluate the entire Fzd expression profile. F9 cells were treated with RA to induce PrE and total RNA was isolated after 3 days and reversetranscribed into cDNA for end point PCR using primers for $F z d 1$ through $F z d 10$, except $F z d 7$ (described later) (Supplementary data). Results showed amplicons for Fzd1, 2, 4, 5, 6, 8 and 10 members in undifferentiated and RA-treated F9 cells (Fig. 1A). The expression of $F z d 3$ and $F z d 9$ 
was not detected even after 40 cycles of PCR or when the annealing temperature was altered (data not shown). To confirm that $F z d 3$ and $F z d 9$ were not expressed in F9 cells, mouse tissues known to express these genes were isolated and used in a PCR analysis with the same primers and conditions used in the F9 analysis. Total RNA was collected from mouse dermis and hippocampus, known to express $F z d 3$ and $F z d 9$, respectively, and reverse-transcribed into cDNA for end point PCR with $F z d 3$ and $F z d 9$ primers (Fig. 1B). $F z d 3$ and $F z d 9$ were expressed in the mouse dermis and hippocampus, respectively, but not in cDNAs from F9 cells. Sequence analysis confirmed the identity of the amplicons as $F z d 3$ and $F z d 9$ (data not shown).

Detecting the expression of these Fzd genes in F9 cells prompted further analysis to determine by quantitative means if the genes were RA-responsive. Since the $F z d$ amplicons shown in Fig. 1 were detected using 40 cycles of PCR, subtle differences in expression of each gene between the undifferentiated and RA-treated cells would be difficult to identify. To address this, F9 cells were treated with DMSO or RA for 3 days and total RNA was isolated and reversetranscribed into cDNA for qRT-PCR. Results showed that $F z d 1$, 4, and 8 were significantly upregulated by RA treatment, showing a 9.82, 4.86, and 3.69-fold increase in expression relative to control, respectively (Fig. 1C). Fzd2 and $F z d 6$ showed a slight increase in expression after exposure to RA, but changes for either were not statistically significant (Fig. 1C). The expression of $F z d 5$ and $F z d 10$ was lower following RA treatment, showing a 0.52 and 0.61 fold-decrease relative to control, respectively, however this decrease in expression was again not statistically significant (Fig. 1C). It appears that while $F z d 1,4$ and 8 genes were RA-responsive, $F z d 2,5,6$, and 10 were not. Furthermore, it is interesting to note that while Fzdl showed the greatest overall increase in this group after three days of RA treatment, $F z d 7$ was the most abundantly expressed of the Fzd family (Fig. 2).

\section{Frizzled 7 expression in undifferentiated F9 and RA-induced PrE cells}

The ability of F9 cells to differentiate into PrE when treated with RA and to PE when exposed subsequently to RA and db-cAMP is well known (Strickland and Mahdavi 1978; Strickland et al. 1980). As noted above, RA-induced differentiation is accompanied by an increase in Wnt6 expression, and WNT6 by itself induces PrE (Krawetz and Kelly 2008). Although a receptor for WNT6 in F9 cells has not been identified, FZD7 is a candidate as there have been several reports linking it and WNT6 (Beaton et al. 2016; Linker et al. 2005; Schmidt 
et al. 2004; Schubert et al. 2002). Furthermore, WNT/ $\beta$-catenin signaling activates TCF/LEF activity in F9 cells (Hwang and Kelly 2012; Sandieson et al. 2014) and there is evidence for TCF/LEF consensus binding sequences in several $F z d$ promoters including $F z d 7$ (Kolben et al. 2012). FZD7 is one of the most studied family member, and is known to bind to several WNT ligands to transduce the signal to a number of different pathways (Phesse et al. 2016). To analyze the expression of $F z d 7$, total RNA was collected from F9 cells treated with RA, and then reversetranscribed into first-strand cDNA for qRT-PCR. The expression profile of Gata6 was first examined to ensure cells had responded to RA by day 4 (Fig. 2A). Gata6 is a RA responsive gene up-regulated when cells differentiate into PrE (Hwang and Kelly 2012), and qRT-PCR analysis showed its expression compared to that in DMSO-treated controls was significantly higher in RA-treated cells (Fig. 2A). Having established that cells had differentiated following RA treatment, the cDNAs collected as described above were analyzed by qRT-PCR and Fzd7specific primers. $F z d 7$ was, however, first examined using end point PCR and results showed the presence of an amplicon in the days 1-4 RA treatments and the DMSO-treated controls (data not shown). These amplicons were sequenced and all were found to be identical to the $F z d 7$ sequence appearing in the NCBI Nucleotide database. qRT- PCR using Fzd7-specific primers showed that there was no significant increase in $F z d 7$ mRNA abundance due to RA treatment on day 3 relative to controls, but the differences were significant by day 4 (Fig. 2B).

\section{Overexpression of $\boldsymbol{F z d} 7$ activates canonical WNT/B-catenin signaling in F9 cells, but is not sufficient to induce PrE}

Up-regulation of $F z d 7$ by RA was originally thought to be an attribute that would contribute to PrE differentiation, but the fact that WNT6-conditioned media differentiates F9 cells to PrE indicated a FZD receptor must be available to tranduce the signal even in the absence of RA (Krawetz and Kelly 2008). Thus, the requirement of $F z d$ genes to be RA responsive may be unnecessary. Nevertheless, given its relatively high level of expression in F9 cells, and the reported link between it and WNT6, Fzd7 was selected for further investigation. There is also precedence for FZD7 as the candidate receptor involved in PrE differentiation, because like WNT6 (Linker et al. 2005; Schmidt et al. 2007), it is involved in the activation of both canonical and non-canonical WNT signaling pathways (Phesse et al. 2016; Zhang et al. 2013). Furthermore, both pathways are necessary for PrE and PE formation, respectively, in F9 
cells (LaMonica et al. 2009; Liu et al. 2002). Fzd7 was the most abundantly expressed $F z d$ in F9 cells, and this is in agreement with the expression profile in human ESCs (Fernandez et al. 2014). Likewise, in NT2/D1 embryonal carcinoma cells several $W n t$ and Fzd genes, including $F z d 7$ are up-regulated in response to RA (Snow et al. 2009). The overexpression of $F z d 7$ has been reported in many types of human cancers, notably, colorectal cancer, hepatocellular carcinoma, and triple-negative breast cancer (Merle et al. 2004; Yang et al. 2011). Previous studies have reported that WNT pathway activation does not occur following the ovexpression of Fzd (Yang et al. 2011), but there is contradictory evidence showing that in the absence of the WNT ligand FZD overexpression can activate the WNT pathway (Sheldahl et al. 1999; Umbhauer et al. 2000; Veeman et al. 2003). If overexpression of $F z d 7$ in F9 cells is sufficient to activate canonical WNT signaling required for PrE formation, then increased $\beta$-catenin/TCFLEF transcriptional activity and downstream targets such as DAB2 should be detected. To test this, F9 cells were transfected with $F z d 7$ or $p c D N A 3.1$ and selected with G418 for 5 days, or treated with RA or DMSO as positive and negative controls for differentiation, respectively. Total RNA was collected, reverse-transcribed into cDNA, and used for qRT-PCR with Fzd7 primers to verify that $F z d 7$ was overexpressed. qRT-PCR results showed that $F z d 7$ mRNA was expressed greater than 50-fold higher in $F z d 7$-transfected cells relative to empty vector control (Fig. 3A). Confident that Fzd7-transfected F9 cells showed increased Fzd7 expression, F9 cells were analyzed for $\beta$-catenin/TCF-LEF-dependent transcription using a Dual-Luciferase reporter assay system. Cells were co-transfected with $p B A R L$ ( $\beta$-catenin-activated reporter luciferase) to detect activation of TCF/LEF-dependent transcription and $p R L-T K$ (Renilla luciferase) plasmids, and either $F z d 7$ or $p c D N A 3.1$-empty vector $(E V)$. Cells co-transfected with $p B A R L$ and $p R L-T K$ and treated with RA or DMSO were used as positive and negative controls, respectively. $p R L-T K$ was used to normalize luciferase values. Relative to the DMSO-treated cells, results indicated that cells treated with RA or transfected with $F z d 7$ showed a significant increase in luciferase activity (4.32 and 3.06-fold higher luciferase activity, respectively) (Fig. 3B). DMSO and $E V$ transfected cells both exhibited approximately equal levels of luciferase activity. Thus, the results indicated that overexpressing $F z d 7$ was sufficient for activation of $\beta$-catenin/TCF-LEFdependent transcription in F9 cells. Surprisingly, however, DAB2 levels were barely detectable in Fzd7-transfected F9 cells, despite the increase in luciferase activity (Fig. 3C). Densitometric analysis further showed that DAB2 levels were not signficantly different in undifferentiated $E V$ - 
tranfected and Fzd7-transfected F9 cells (Fig. 3D). Furthermore, TROMA-1 signals, which would denote cytokeratin 8 in differentiated cells (Brulet et al. 1980) were only seen in the RApositive controls (Fig. 3E). Although there may be several reasons to explain these results, it is likely that despite the reporter assay being sensitive enough to indicate activation, the overexpression by itself was not adequate for the requisite activation of endogenous genes needed to induce these naïve cells. However, without demonstrating increased FZD7 protein expression in transfected cells, alternative explanations for the discrepancy between increased reporter activity in the absence of differentiation must also be considered.

\section{Fzd7 knockdown does not inhibit RA-induced primitive endoderm differentiation}

Knockdown of $F z d 7$ in many human cancer cell types decreases $\beta$-catenin/TCF-LEF activity, invasion, and migration (Ueno et al. 2009; Yang et al. 2011). If this is true for F9 cells, and FZD7 binds WNT6 to transduce a differentiation signal, then depleting cells of $F z d 7$ is expected to prevent PrE differentiation. To address if F9 cells under reduced levels of $F z d 7$ could be induced to differentiate into PrE, cells were transfected with short interfering RNA (siRNA) to knock down endogenous $F z d 7$ transcripts or transfected with a non-targeting control siRNA. qRT-PCR was used to confirm that $F z d 7$ mRNA levels were reduced in $F z d 7$ siRNA-transfected cells relative to the non-targeting control siRNA-transfected cells (Fig. 4A). Given these results and confident that this approach had reduced $F z d 7$ expression, siRNA-transfected cells were treated with RA and protein lysates were harvested for immunoblot analysis using DAB2 and TROMA-1 antibodies. Immunoblot and densitometric analyses showed that DAB2 levels were significantly less in Fzd7 siRNAs-transfected F9 cells when compared to non-targeting control cells treated with RA (Figs. 4B and 4C, respectively). Cytokeratin8 positive intermediate filaments, however, were present at approximately equal amounts in all RA-treated cells, including those transfected with $F z d 7$ siRNAs or non-targeting control siRNA (Fig. 4D). Densitometric analysis further showed that TROMA-1 levels were not significantly different due to $F z d 7$ siRNA transfection (Fig. 4E). These results indicated that reducing the message encoding the FZD7 receptor using an siRNA approach reduced DAB2 signal, but did not have a detrimental effect on the appearance of TROMA-1 intermediate filaments indicative of $\operatorname{PrE}$ differentiation. Thus, despite the knockdown of $F z d 7$, which should have reduced the overall 
amount of the protein, the remaining may be still sufficient to transduce the WNT6 signal. Since FZD7 protein levels were not assessed in this study due to inherent specificity problems associated with commercially-available antibodies, others scenarios may exist. For instance, these results would not support the idea that FZD7 is the receptor necessary for PrE differentiation, but given the functional redundacy issue reported for FZD receptors (Huang and Klein 2004), however, it is also possible that reduced levels of the FZD7 receptor were compensated by one or more FZDs, encoded by mRNAs already present in F9 cells (see above). Without reliable antibodies to the FZD family or the ability to knock-down the expression of all $F z d$ genes, and then rescue each on an individual basis, this would be difficult to test. Homology analysis of FZD1, 2 and 7 indicates that they share approximately $75 \%$ sequence identity, and even though this number falls between FZDs in different clusters, there is still a significant amount of overlap (Fredriksson et al. 2003). For example, FZD5 and FZD7, which are in different clusters, are functionally redundant for canonical WNT/ $\beta$-catenin signaling in cell culture, as ectopic expression of $F z d 5$ in human mesenchymal stem cells (hMSCs) harboring a $F z d 7$ knockdown can rescue TCF/LEF luciferase reporter activity. Similarly, ectopic expression of $F z d 7$ can rescue the same reporter activity in Fzd5 knockdown hMSCs (Kolben et al. 2012). Given this information, an alternative strategy would be to knockdown more than one FZD receptor, and if even technically plausible, the possibility remains that another Fzd could be upregulated in response. Unfortunately, and despite the fact that there appear to be more reports detailing the use of antibodies to inhibit FZDs (Blagodatski et al. 2014), and specifically FZD7 (Ueno et al. 2009; Ueno et al. 2013), and in light of the paucity of direct evidence in assigning individual FZDs to specific WNTs (Dijksterhuis et al. 2015; Dijksterhuis et al. 2014), testing each scenario would be a laborious process.

Although the knockdown of $F z d 7$ in F9 cells did not attenuate RA-induced PrE differentiation, given that Wnt6 expression is down-regulated when PrE cells are treated with dbcAMP (Krawetz and Kelly 2008), and knowing mechanisms are in place to temper canonical WNT signaling during differentiation (Schlupf and Steinbeisser 2014; Shibamoto et al. 2004) we investigated how canonical WNT6 signaling might be involved in this attenuation process.

\section{Dkk1 mRNA expression is induced by RA treatment and Wnt6 overexpression}

A microarray profiling study using F9 cells reported on two RA-target genes Dab2 and sFRP5, which encode inhibitors of WNT signaling (Zhuang et al. 2003). Interestingly, F9 cells 
induced to differentiate into VE are not responsive to WNT ligands, pointing to a blockade in WNT signaling that accompanies differentiation (Shibamoto et al. 2004). Another defined mechanism involves IGF, which induces Axin2 and stabilizes AXIN1 (Schlupf and Steinbeisser 2014). Thus, there are several mechanisms in place, but other inhibitors of WNT signaling may also be induced in response to activated WNT6 signaling. Candidates that would serve in this capacity to antagonize WNT- $\beta$-catenin signaling are members of the DKK family, which are upregulated in F9 cells induced to form VE (Shibamoto et al. 2004). Furthermore, DKK1/Dkk1 is a direct target gene of WNT- $\beta$-catenin signaling as several TCF-binding element consensus sites have been identified within a highly conserved region $1 \mathrm{~kb}$ upstream of the transcription start site in the human and mouse promoters (Niehrs 2006; Niida et al. 2004). Other evidence comes from studies showing that DKK1 acts as a secreted antagonist specifically inhibiting canonical WNT signaling when it binds to the LDL receptor-related protein 5/6 (LRP5/6) co-receptor, which blocks the LRP5/6-WNT interaction that is obligatory for canonical pathway activation (He et al. 2004; Liu et al. 2002; Pinson et al. 2000). Therefore, to test if Dkkl was up-regulated in PrE cells, F9 cells were treated with $10^{-7} \mathrm{M}$ RA for 1-4 days, or DMSO for 4 days and total RNA was collected and reverse-transcribed into cDNA for qRT-PCR analysis (Fig. 5). Results indicated that $D k k 1$ expression increased by 118 and 611-fold in cells treated with RA for 3 and 4 days, respectively, compared to DMSO control cells (Fig. 5A). Thus, the Dkkl gene like Wnt6 was RA-responsive, but whether or not it is a WNT6 target gene required further analysis.

F9 cells were transfected with Wnt6 or $p c D N A 3.1-E V$, and then treated with G418 for 4 days, and total RNA was collected and reverse-transcribed into cDNA to be used as template for end point and qRT-PCR analyses. To confirm that Wnt6 was being overexpressed, cDNAs were used for end point PCR with primers for Wnt6. Results showed the presence of a Wnt6 amplicon in cells transfected with pcDNA3.1-Wnt6 (Fig. 5B). Correspondingly, a Wnt6 amplicon was not detected in cDNA from empty vector-transfected cells, but the presence of an $L 14$ amplicon indicated that cDNA was available for amplification. Confident that Wnt6 was being overexpressed, $D k k 1$ expression was examined and results showed a 2.92-fold increase in $D k k 1$ expression in cells transfected with Wnt6 compared to the empty vector-transfected controls (Fig. 5C).

$D K K 1 / D k k 1$ is reportedly regulated by the $\beta$-catenin/TCF/LEF transcriptional complex in several cell lines, and there are four TCF/LEF DNA binding motifs located within the human 
(DKK1) and mouse $D k k 1$ promoters (Logan and Nusse 2004). Since Dkkl expression was greatly increased in the presence of RA, and only slightly increased due to Wnt6 overexpression, subsequent analysis focussed on searching the mouse $D k k 1$ promoter region for conserved Retinoic Acid Response Elements (RARE) binding sites, the presence of which might explain the greater than 600-fold upregulation of $D k k 1$ by RA. The Ensembl Genome Browser Regulatory Build was used to identify putative regulatory regions upstream of the $D k k 1$ transcription start site, and a 961 bp sequence was predicted as the $D k k 1$ promoter region. This sequence was used to identify putative RARE consensus DNA motifs and two conserved RARE nucleotide sequences were found within this region, spaced 9bp apart (data not shown). These in silico results would suggest that in addition to Dkk1 as being a WNT6 target gene, the potential exists that the regulation of the $D k k 1$ is also under the control of RAR-RXR homo/heterodimers. ChIP analysis should provide more insight into the $D k k 1$ regulation by WNT- $\beta$-catenin and RA signaling, and these studies are currently underway.

Since $D k k 1$ expression was up-regulated in response to RA and Wnt6 overexpression, and overwhelming evidence indicates that DKK1 is a WNT antagonist, then the ectopic expression of $D k k 1$ should block PrE differentiation. To test this, F9 cells were transfected with a Myc-tagged human DKK1 (Myc-DKK1) construct and selected with G418, then treated with RA for 4 days to induce PrE. Protein lysates were collected and processed for immunoblot analysis with the TROMA-1 antibody to determine if cells had been blocked from differentiating into PrE. Immunoblot analysis showed that cytokeratin 8 was expressed in the positive controls treated with RA or transfected with empty vector and treated with RA (Fig. 6A). Cytokeratin 8 levels, however, appeared to be reduced in cells transfected with the $M y c-D K K 1$ construct and treated with RA (Fig. 6A). Densitometric analysis confirmed this significant decrease in TROMA-1 levels in cells transfected with $M y c-D K K 1$ and treated with RA compared to cells transfected with empty vector and treated with RA (Fig. 6B). The reduction in the TROMA-1 signal in cells transfected with empty vector and treated with RA compared to cells treated with RA alone would indicate that the transfection had affected differentiation (Fig. 6A). Although this should not have occurred, all subsequent experiments were conducted using transfected-positive $(p c D N A 3.1+\mathrm{RA})$ and negative controls ( $p c D N A 3.1+\mathrm{DMSO})$. Experiments were repeated to examine another marker of PrE differentiation and intracellular WNT antagonist, encoded by Dab2 (Cho et al. 1999; Zhuang et al. 2003), which when translated inhibits WNT/ $\beta$-catenin 
signaling by binding LRP6 and promoting its internalization, as well as by interacting with and stabilizing AXIN, preventing the destruction complex disassembly (Jiang et al. 2008; Pataki et al. 2015). If $M y c-D K K 1$ ectopic expression negatively affected PrE differentiation, then it was expected that Dab2 expression would be less than the controls. Total RNA was collected and reverse-transcribed into cDNA and used for qRT-PCR analysis with Dab2 primers. Results showed that relative to controls, Dab2 was upregulated more than 140-fold in empty vectortransfected and RA-treated cells, and by nearly 120-fold in cells transfected with Myc-DKK1 and treated with RA (Fig. 6C). The difference was significant, and indicated that ectopically expressed $M y c-D K K 1$ in undifferentiated F9 cells reduced their ability to differentiate into PrE when treated with RA.

The results from the overexpression of $M y c-D K K 1$ indicated that ectopically expressing a construct encoding a secreted antagonist of canonical WNT/ $\beta$-catenin signaling in undifferentiated F9 cells attenuated the ability of RA to induce the PrE phenotype. To corroborate this data, conditioned medium (CM) from HEK-293 cells expressing Myc-DKK1 was applied to F9 cells prior to being treated with RA. Briefly, HEK-293 cells were transfected with $p c D N A 3.1$ or $M y c-D K K 1$, and media was collected after 48 hours, filter sterilized, and concentrated. HEK-293 protein lysates were also collected for immunoblot analysis with the anti-c-MYC antibody (9E10) to confirm that the cells were translating the Myc-DKK1 transcripts. A signal at approximately $39 \mathrm{kD}$ corresponding to the molecular mass of MYCDKK1 was detected in HEK-293 cells transfected with the Myc-DKK1 construct, but not in cells transfected with the empty vector (Fig. 7A). To confirm that the HEK-293 cells were secreting the protein, the CM generated from these cells was concentrated and used for immunoblot analysis. If the MYC-DKK1 protein was being translated and secreted by the cells, then the 9E10 antibody should recognize the MYC-epitope both in cell lysates and in the CM. The concentrated CM was used for immunoblot analysis and a $39 \mathrm{kD}$ signal was detected in the medium from cells transfected with $M y c-D K K 1$, but not the empty vector (data not shown). Having evidence that MYC-DKK1 was present in the CM, it was applied to F9 cells in the presence of DMSO or RA to determine if it would mimic the effect of ectopically expressing $M y c-D K K 1$ on differentiation. Protein lysates were collected for immunoblot analysis and results showed a TROMA-1 signal present in cells treated with the empty vector-CM and RA, but absent in cells treated with this CM and DMSO. Thus, the CM did not contain factors that induced differentiation on its own 
(Fig. 7B). A TROMA-1 signal was present in cells treated with MYC-DKK1 CM and treated with RA, however, its intensity appeared to be reduced compared to that seen in cells treated with the empty vector-CM and RA (Fig. 7B). Densitometric analysis confirmed that the reduction of TROMA-1 seen in cells treated with MYC-DKK1 CM and RA was significant compared to cells treated with the empty vector-CM and RA (Fig. 7C). Taken together, these results indicated that the HEK-293 cells were a useful vehicle for the production and secretion of the MYC-DKK1 protein, and more importantly applying this CM to F9 cells in the presence of RA attenuated the ability of the latter to induce PrE. Thus, and as noted above, the blockade in WNT sginaling required for cells to form VE (Shibamoto et al. 2004), occurs much earlier, at the time when cells have completed their differentiation to PrE. Furthermore, these results demonstrated that the $D k k 1$ gene was induced by RA treatment, and more importantly it is a WNT6 target gene. This expression pattern of $D k k 1$ parallels that of another inhibitor of WNT signaling, Dab2, suggesting that canonical WNT/ $\beta$-catenin signaling is inhibited in PrE cells at more than one level and is the result of a negative feedback loop initiated by WNT6.

In conclusion, we have shown that eight of ten $F z d$ genes were expressed in undifferentiated F9 cells and only three were RA-regulated (Fig. 1). Fzd7 is the most highly expressed of the Fzd genes expresssed in F9 cells (Fig. 2), and is up-regulated by RA (Fig. 2B). Although the overexpression of $F z d 7$ was sufficient to activate a WNT- $\beta$-catenin reporter, it was not sufficient to increase DAB2 levels or induce PrE formation (Fig. 3). However, knocking down $F z d 7$ transcripts attenuated DAB2 levels, but had no apparent effect on differentiation (Fig. 4). Based on the data, we cannot definitely conclude that FZD7 is the bona fide WNT6 receptor, as the possibility exists that the knockdown was compensated by another FZD receptor acting in a functionally redundant manner, and/or if the overexpression experiments were ineffective in producing enough protein necesssary to induce differentiation. However, we have identified a WNT6-responsive gene encoding a WNT antagonist, DKK1 (Fig. 5) known to particate in the differentiation of extraembryonic endoderm (Shibamoto et al. 2004). In this model, canonical WNT6 signaling in F9 cells is necessary and sufficient for PrE formation, and in a feedforward manner the subsequent WNT-induced antagonism between the ligand and the FZD receptor(s) by DKK1, is necessary, as others have reported (LaMonica et al. 2009; Schlupf and Steinbeisser 2014), for extraembryonic endoderm differentiation to occur (Figs. 6 and 7). Our model also predicts that although Wnt6 expression is down-regulated in PrE cells (Krawetz 
and Kelly 2008), canonical WNT signaling is eventually blocked in part following the translation of mRNAs encoding antagonists like DAB2 (Zhuang et al. 2003) and DKK1 (this study). Since DKK1 antagonizes canonical WNT signaling by binding the WNT LRP5/6 co-receptor (Pinson et al. 2000), any remaining WNT6 would still be available to bind other FZDs and/or coreceptors to activate non-canonical, $\beta$-catenin independent WNT signaling required for extraembryonic endoderm to form (Bikkavilli et al. 2008; LaMonica et al. 2009). There is precedence for the ability of the same WNT to activate canonical $\beta$-catenin-dependent signaling over the non-canonical pathways and vice versa depending on the presesence of specific cofactors and/or co-recptors (Beaton et al. 2016; Mikels and Nusse 2006; Schmidt et al. 2007; Yamamoto et al. 2008). Thus, it is tempting to speculate that F9 cells also utilize a similar mechanism that involves DKK1 and other inhibitors to help mediate a switch from canonical WNT/ $\beta$-catenin to non-canonical PCP signaling in order to facilitate the transition from PrE to PE. This model is currently being investigated as it will help further the understanding of how WNTs preferentially choose to activate specific downstream signaling pathways.

\section{Acknowledgements}

GMK would like to thank the Natural Sciences and Engineering Research Council of Canada for funding. The authors acknowledge the Faculty of Graduate and Postgraduate Studies, Western University for support to GG and MIG, and they would like to thank members of the Kelly lab for suggestions and comments throughout the course of this study.

\section{References}

Beaton, H., Andrews, D., Parsons, M., Murphy, M., Gaffney, A., Kavanagh, D., McKay, G.J., Maxwell, A.P., Taylor, C.T., Cummins, E.P., Godson, C., Higgins, D.F., Murphy, P., and Crean, J. 2016. Wnt6 regulates epithelial cell differentiation and is dysregulated in renal fibrosis. Am J Physiol Renal Physiol: ajprenal 00136 02016. doi: 10.1152/ajprenal.00136.2016.

Bikkavilli, R.K., Feigin, M.E., and Malbon, C.C. 2008. G alpha o mediates WNT-JNK signaling through dishevelled 1 and 3, RhoA family members, and MEKK 1 and 4 in mammalian cells. J Cell Sci 121(Pt 2): 234-245. doi: 10.1242/jcs.021964. 
Blagodatski, A., Poteryaev, D., and Katanaev, V.L. 2014. Targeting the Wnt pathways for therapies. Mol Cell Ther 2: 28. doi: 10.1186/2052-8426-2-28.

Boiani, M., and Scholer, H.R. 2005. Regulatory networks in embryo-derived pluripotent stem cells. Nat Rev Mol Cell Biol 6(11): 872-884. doi: 10.1038/nrm1744.

Brulet, P., Babinet, C., Kemler, R., and Jacob, F. 1980. Monoclonal antibodies against trophectoderm-specific markers during mouse blastocyst formation. Proc Natl Acad Sci U S A 77(7): 4113-4117.

Cho, S.Y., Cho, S.Y., Lee, S.H., and Park, S.S. 1999. Differential expression of mouse Disabled 2 gene in retinoic acid-treated F9 embryonal carcinoma cells and early mouse embryos. Mol Cells 9(2): 179-184.

Dijksterhuis, J.P., Baljinnyam, B., Stanger, K., Sercan, H.O., Ji, Y., Andres, O., Rubin, J.S., Hannoush, R.N., and Schulte, G. 2015. Systematic mapping of WNT-FZD protein interactions reveals functional selectivity by distinct WNT-FZD pairs. J Biol Chem 290(11): 6789-6798. doi: 10.1074/jbc.M114.612648.

Dijksterhuis, J.P., Petersen, J., and Schulte, G. 2014. WNT/Frizzled signalling: receptor-ligand selectivity with focus on FZD-G protein signalling and its physiological relevance: IUPHAR Review 3. Br J Pharmacol 171(5): 1195-1209. doi: 10.1111/bph.12364.

Fernandez, A., Huggins, I.J., Perna, L., Brafman, D., Lu, D., Yao, S., Gaasterland, T., Carson, D.A., and Willert, K. 2014. The WNT receptor FZD7 is required for maintenance of the pluripotent state in human embryonic stem cells. Proc Natl Acad Sci U S A 111(4): 14091414. doi: 10.1073/pnas.1323697111.

Fredriksson, R., Lagerstrom, M.C., Lundin, L.G., and Schioth, H.B. 2003. The G-proteincoupled receptors in the human genome form five main families. Phylogenetic analysis, paralogon groups, and fingerprints. Mol Pharmacol 63(6): 1256-1272. doi: 10.1124/mol.63.6.1256.

He, X., Semenov, M., Tamai, K., and Zeng, X. 2004. LDL receptor-related proteins 5 and 6 in Wnt/beta-catenin signaling: arrows point the way. Development 131(8): 1663-1677. doi: 10.1242/dev.01117.

Huang, H.C., and Klein, P.S. 2004. The Frizzled family: receptors for multiple signal transduction pathways. Genome Biol 5(7): 234. doi: 10.1186/gb-2004-5-7-234.

Hwang, J.T., and Kelly, G.M. 2012. GATA6 and FOXA2 regulate Wnt6 expression during extraembryonic endoderm formation. Stem Cells Dev 21(17): 3220-3232. doi: 10.1089/scd.2011.0492.

Jiang, Y., Prunier, C., and Howe, P.H. 2008. The inhibitory effects of Disabled-2 (Dab2) on Wnt signaling are mediated through Axin. Oncogene 27(13): 1865-1875. doi:

10.1038/sj.onc.1210829.

Kawano, Y., and Kypta, R. 2003. Secreted antagonists of the Wnt signalling pathway. J Cell Sci 116(Pt 13): 2627-2634. doi: 10.1242/jcs.00623.

King, T.D., Zhang, W., Suto, M.J., and Li, Y. 2012. Frizzled7 as an emerging target for cancer therapy. Cell Signal 24(4): 846-851. doi: 10.1016/j.cellsig.2011.12.009.

Kolben, T., Perobner, I., Fernsebner, K., Lechner, F., Geissler, C., Ruiz-Heinrich, L., Capovilla, S., Jochum, M., and Neth, P. 2012. Dissecting the impact of Frizzled receptors in Wnt/beta-catenin signaling of human mesenchymal stem cells. Biol Chem 393(12): 14331447. doi: 10.1515/hsz-2012-0186. 
Krawetz, R., and Kelly, G.M. 2008. Wnt6 induces the specification and epithelialization of F9 embryonal carcinoma cells to primitive endoderm. Cell Signal 20(3): 506-517. doi: 10.1016/j.cellsig.2007.11.001.

LaMonica, K., Bass, M., and Grabel, L. 2009. The planar cell polarity pathway directs parietal endoderm migration. Dev Biol 330(1): 44-53. doi: 10.1016/j.ydbio.2009.03.008.

Linker, C., Lesbros, C., Gros, J., Burrus, L.W., Rawls, A., and Marcelle, C. 2005. beta-Catenindependent Wnt signalling controls the epithelial organisation of somites through the activation of paraxis. Development 132(17): 3895-3905. doi: 10.1242/dev.01961.

Liu, T., Lee, Y.N., Malbon, C.C., and Wang, H.Y. 2002. Activation of the beta-catenin/Lef-Tcf pathway is obligate for formation of primitive endoderm by mouse F9 totipotent teratocarcinoma cells in response to retinoic acid. J Biol Chem 277(34): 30887-30891. doi: 10.1074/jbc.M203852200.

Logan, C.Y., and Nusse, R. 2004. The Wnt signaling pathway in development and disease. Annu Rev Cell Dev Biol 20: 781-810. doi: 10.1146/annurev.cellbio.20.010403.113126.

Merle, P., de la Monte, S., Kim, M., Herrmann, M., Tanaka, S., Von Dem Bussche, A., Kew, M.C., Trepo, C., and Wands, J.R. 2004. Functional consequences of frizzled-7 receptor overexpression in human hepatocellular carcinoma. Gastroenterology 127(4): 1110-1122.

Mikels, A.J., and Nusse, R. 2006. Purified Wnt5a protein activates or inhibits beta-catenin-TCF signaling depending on receptor context. PLoS Biol 4(4): e115. doi: 10.1371/journal.pbio.0040115.

Moon, R.T. 2005. Wnt/beta-catenin pathway. Sci STKE 2005(271): cm1. doi: $10.1126 /$ stke. $2712005 \mathrm{~cm} 1$.

Niehrs, C. 2006. Function and biological roles of the Dickkopf family of Wnt modulators. Oncogene 25(57): 7469-7481. doi: 10.1038/sj.onc. 1210054.

Niida, A., Hiroko, T., Kasai, M., Furukawa, Y., Nakamura, Y., Suzuki, Y., Sugano, S., and Akiyama, T. 2004. DKK1, a negative regulator of Wnt signaling, is a target of the betacatenin/TCF pathway. Oncogene 23(52): 8520-8526. doi: 10.1038/sj.onc.1207892.

Okoye, U.C., Malbon, C.C., and Wang, H.Y. 2008. Wnt and Frizzled RNA expression in human mesenchymal and embryonic (H7) stem cells. J Mol Signal 3: 16. doi: 10.1186/17502187-3-16.

Pataki, C.A., Couchman, J.R., and Brabek, J. 2015. Wnt Signaling Cascades and the Roles of Syndecan Proteoglycans. J Histochem Cytochem. doi: 10.1369/0022155415586961.

Phesse, T., Flanagan, D., and Vincan, E. 2016. Frizzled7: A Promising Achilles' Heel for Targeting the Wnt Receptor Complex to Treat Cancer. Cancers (Basel) 8(5). doi: 10.3390/cancers8050050.

Pinson, K.I., Brennan, J., Monkley, S., Avery, B.J., and Skarnes, W.C. 2000. An LDL-receptorrelated protein mediates Wnt signalling in mice. Nature 407(6803): 535-538. doi: $10.1038 / 35035124$.

Rossant, J., and Tam, P.P. 2009. Blastocyst lineage formation, early embryonic asymmetries and axis patterning in the mouse. Development 136(5): 701-713. doi: 10.1242/dev.017178.

Sandieson, L., Hwang, J.T., and Kelly, G.M. 2014. Redox regulation of canonical Wnt signaling affects extraembryonic endoderm formation. Stem Cells Dev 23(10): 1037-1049. doi: $10.1089 / \mathrm{scd} .2014 .0010$.

Schlupf, J., and Steinbeisser, H. 2014. IGF antagonizes the Wnt/beta-Catenin pathway and promotes differentiation of extra-embryonic endoderm. Differentiation 87(5): 209-219. doi: 10.1016/j.diff.2014.07.003. 
Schmidt, C., McGonnell, I.M., Allen, S., Otto, A., and Patel, K. 2007. Wnt6 controls amniote neural crest induction through the non-canonical signaling pathway. Dev Dyn 236(9): 2502-2511. doi: 10.1002/dvdy.21260.

Schmidt, C., Stoeckelhuber, M., McKinnell, I., Putz, R., Christ, B., and Patel, K. 2004. Wnt 6 regulates the epithelialisation process of the segmental plate mesoderm leading to somite formation. Dev Biol 271(1): 198-209. doi: 10.1016/j.ydbio.2004.03.016.

Schubert, F.R., Mootoosamy, R.C., Walters, E.H., Graham, A., Tumiotto, L., Munsterberg, A.E., Lumsden, A., and Dietrich, S. 2002. Wnt6 marks sites of epithelial transformations in the chick embryo. Mech Dev 114(1-2): 143-148.

Sheldahl, L.C., Park, M., Malbon, C.C., and Moon, R.T. 1999. Protein kinase C is differentially stimulated by Wnt and Frizzled homologs in a G-protein-dependent manner. Curr Biol 9(13): 695-698.

Shibamoto, S., Winer, J., Williams, M., and Polakis, P. 2004. A blockade in Wnt signaling is activated following the differentiation of F9 teratocarcinoma cells. Exp Cell Res 292(1): 11-20.

Snow, G.E., Kasper, A.C., Busch, A.M., Schwarz, E., Ewings, K.E., Bee, T., Spinella, M.J., Dmitrovsky, E., and Freemantle, S.J. 2009. Wnt pathway reprogramming during human embryonal carcinoma differentiation and potential for therapeutic targeting. BMC Cancer 9: 383. doi: 10.1186/1471-2407-9-383.

Strickland, S., and Mahdavi, V. 1978. The induction of differentiation in teratocarcinoma stem cells by retinoic acid. Cell 15(2): 393-403.

Strickland, S., Smith, K.K., and Marotti, K.R. 1980. Hormonal induction of differentiation in teratocarcinoma stem cells: generation of parietal endoderm by retinoic acid and dibutyryl cAMP. Cell 21(2): 347-355.

Ueno, K., Hazama, S., Mitomori, S., Nishioka, M., Suehiro, Y., Hirata, H., Oka, M., Imai, K., Dahiya, R., and Hinoda, Y. 2009. Down-regulation of frizzled-7 expression decreases survival, invasion and metastatic capabilities of colon cancer cells. Br J Cancer 101(8): 1374-1381. doi: 10.1038/sj.bjc.6605307.

Ueno, K., Hirata, H., Hinoda, Y., and Dahiya, R. 2013. Frizzled homolog proteins, microRNAs and Wnt signaling in cancer. Int J Cancer 132(8): 1731-1740. doi: 10.1002/ijc.27746.

Umbhauer, M., Djiane, A., Goisset, C., Penzo-Mendez, A., Riou, J.F., Boucaut, J.C., and Shi, D.L. 2000. The C-terminal cytoplasmic Lys-thr-X-X-X-Trp motif in frizzled receptors mediates Wnt/beta-catenin signalling. EMBO J 19(18): 4944-4954. doi: 10.1093/emboj/19.18.4944.

Veeman, M.T., Axelrod, J.D., and Moon, R.T. 2003. A second canon. Functions and mechanisms of beta-catenin-independent Wnt signaling. Dev Cell 5(3): 367-377.

Verheijen, M.H., Wolthuis, R.M., Bos, J.L., and Defize, L.H. 1999. The Ras/Erk pathway induces primitive endoderm but prevents parietal endoderm differentiation of F9 embryonal carcinoma cells. J Biol Chem 274(3): 1487-1494.

Yamamoto, S., Nishimura, O., Misaki, K., Nishita, M., Minami, Y., Yonemura, S., Tarui, H., and Sasaki, H. 2008. Cthrc1 selectively activates the planar cell polarity pathway of Wnt signaling by stabilizing the Wnt-receptor complex. Dev Cell 15(1): 23-36. doi: 10.1016/j.devcel.2008.05.007.

Yang, L., Wu, X., Wang, Y., Zhang, K., Wu, J., Yuan, Y.C., Deng, X., Chen, L., Kim, C.C., Lau, S., Somlo, G., and Yen, Y. 2011. FZD7 has a critical role in cell proliferation in triple negative breast cancer. Oncogene 30(43): 4437-4446. doi: 10.1038/onc.2011.145. 
Zhang, Z., Rankin, S.A., and Zorn, A.M. 2013. Different thresholds of Wnt-Frizzled 7 signaling coordinate proliferation, morphogenesis and fate of endoderm progenitor cells. Dev Biol 378(1): 1-12. doi: 10.1016/j.ydbio.2013.02.024.

Zhuang, Y., Faria, T.N., Chambon, P., and Gudas, L.J. 2003. Identification and characterization of retinoic acid receptor beta2 target genes in F9 teratocarcinoma cells. Mol Cancer Res 1(8): 619-630. 
A
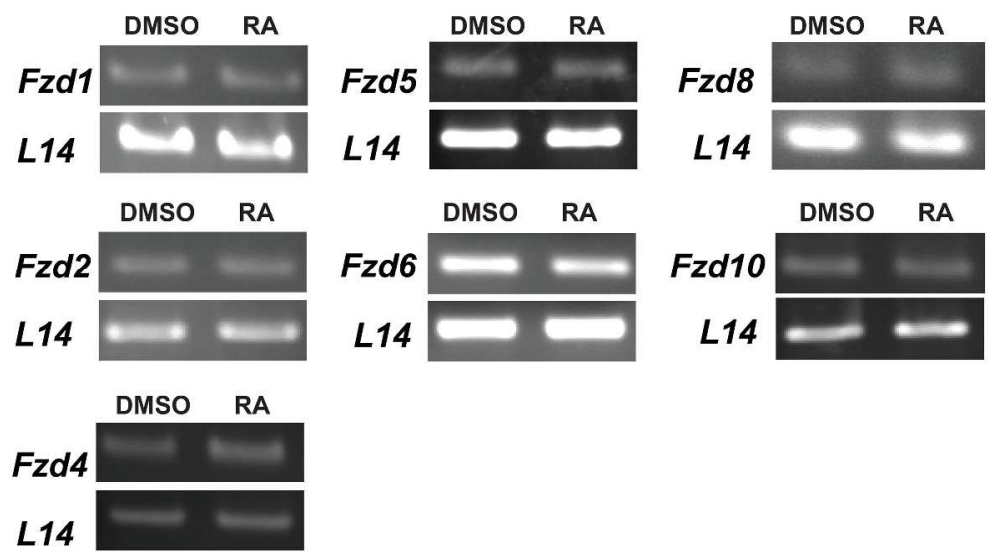

B

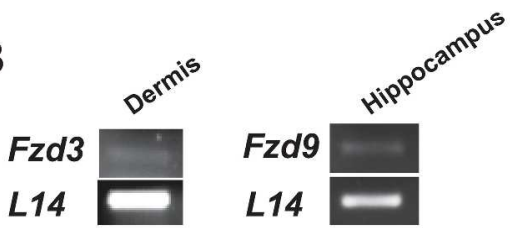

C

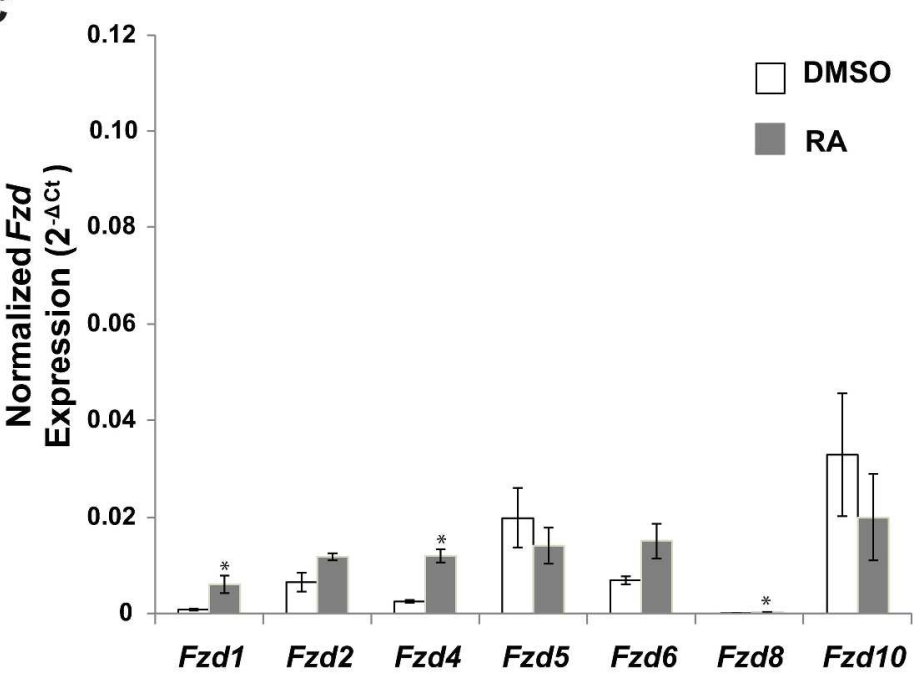

Fig. $1 \mathrm{Fzd}$ gene expression in F9 cells. Total RNA was collected from F9 cells treated with either RA or DMSO for 3 days or from mouse dermis and hippocampus and used as template for RT-PCR. (A) Agarose gels showing amplicons corresponding to the predicted size of each Fzd amplicon. (B) Fzd3 and Fzd9 amplicons were detected in mouse dermal and hippocampal tissues, confirming the utility of the primers used in the F9 cell analysis. Data are representative of three independent experiments. (C) Fzd receptor expression (excluding $F z d 7$ ) in response to RA treatment. qRT-PCR analysis was used to determine the gene expression profile of $F z d$ receptors relative to the control gene $L 14$ in response to RA. While Fzd1, 4 and 8 were significantly upregulated by RA at 3 days $(9.82,4.86$, and 3.69 fold up-regulation relative to DMSO control cells, respectively; Supplementary data), Fzd2, 5, 6, and 10 did not change significantly. Asterisks denote significant difference $(p \leq 0.05)$. Data represent the mean and standard error of three independent experiments. Fold changes in Fzd mRNA expression relative to DMSO are presented in the Supplementary data. 
$261 \times 383 \mathrm{~mm}(300 \times 300$ DPI) 
A

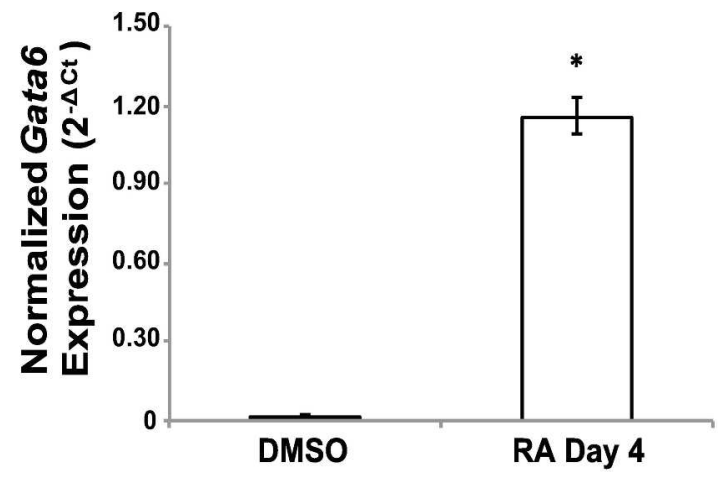

B
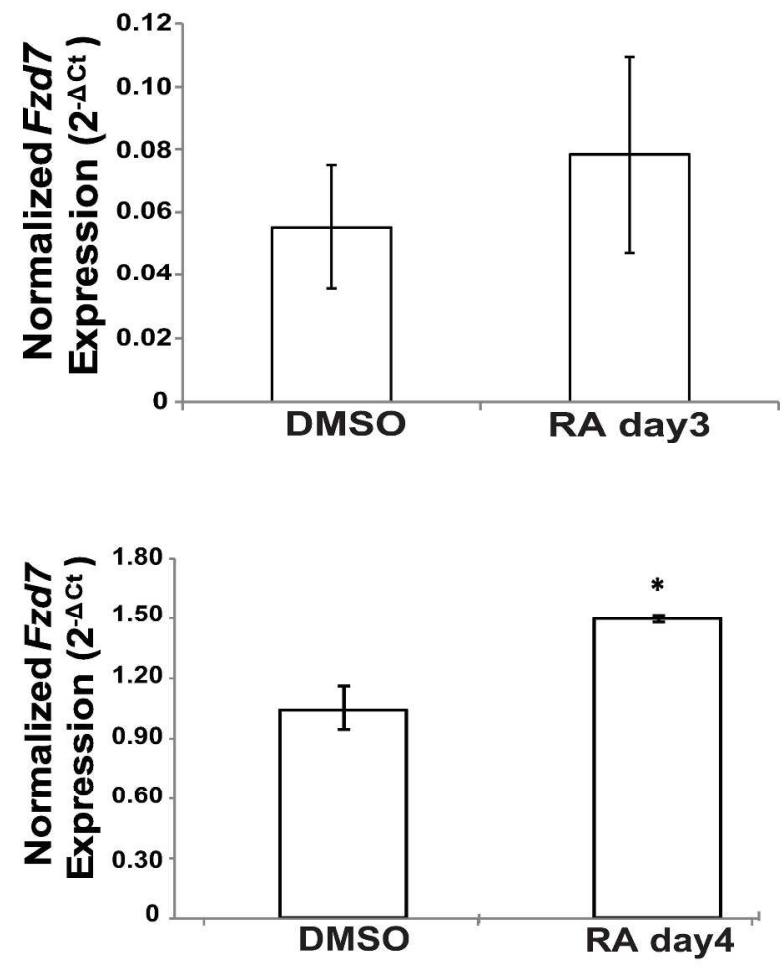

Fig. 2. Fzd7 expression in undifferentiated, and PrE cells. (A) qRT-PCR results showing the dramatic increase in Gata6 expression in F9 cells following RA treatment. (B) qRT-PCR analysis of Fzd7 in cells treated with RA for 4 days, but not 3 days, shows that $F z d 7$ expression was significantly higher when compared to undifferentiated (DMSO-treated) F9 cells. Data are representative of three independent experiments. *denotes significance $(p<0.05)$.

$226 \times 365 \mathrm{~mm}(300 \times 300$ DPI $)$ 
A

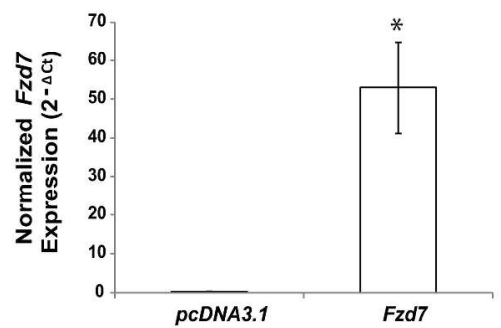

C

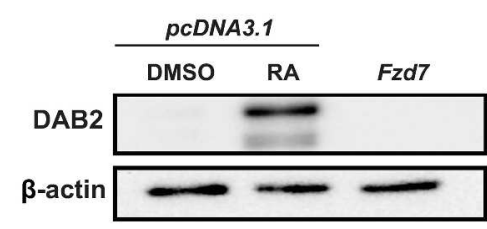

E
B

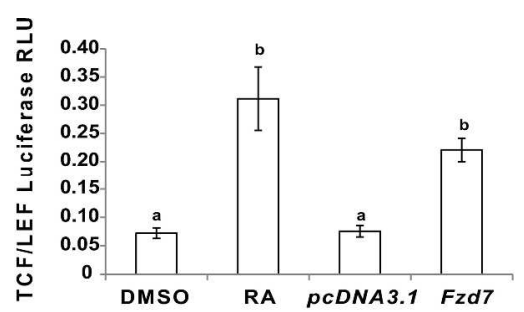

D

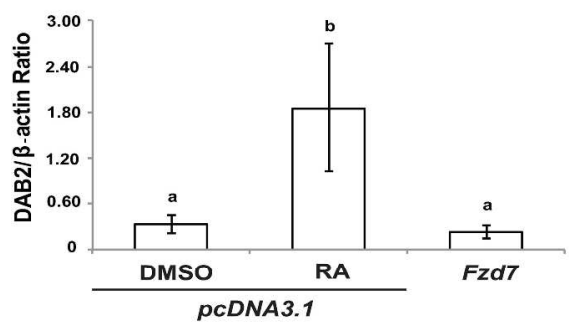

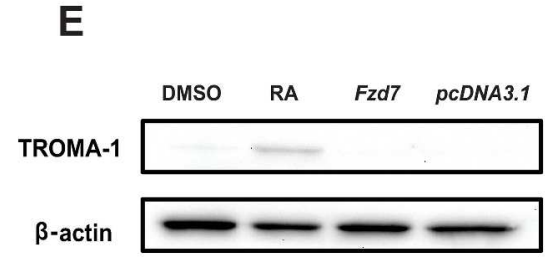

Fig. 3. Overexpression of Fzd7 was sufficient to activate $\beta$-catenin/TCF/LEF-dependent transcription in F9 cells, but not at inducing differentiation. (A) F9 cells were transfected with pcDNA3.1 or Fzd7, or treated with DMSO or RA and total RNA or protein lysate was collected after 5 days. qRT-PCR analysis with primers for Fzd7 showed that Fzd7 mRNA was expressed 163-fold higher in Fzd7-transfected cells relative to pcDNA3.1-transfected cells. (B) F9 cells were co-transfected with $p B A R L$ ( $\beta$-catenin activated reporter luciferase) and $p R L-T K$ (Renilla luciferase) plasmids, as well as either Fzd7 or pcDNA3.1-empty vector, or treated with RA or DMSO for $48 \mathrm{~h}$. Luciferase activity results showed that cells treated with RA or Fzd7

showed a 4.32 and a 3.06 fold-increase in activity, respectively, relative to DMSO-treated cells (Supplementary data). (C) Representative blot showing DAB2 signals in RA-treated F9 cells, but not in DMSO-treated or Fzd7-transfected cells. (D) Densitometric analysis confirmed that DAB2 levels were significantly different in RA-treated F9 cells, but not in DMSO-treated or Fzd7-transfected cells. (E) Representative blot showing a TROMA-1 signal in RA-treated, but not in DMSO-treated, Fzd7 or pcDNA3.1transfected cells. Antibodies against $\beta$-actin were used to ensure equal loading in all lanes. Data represent 
the mean and standard error of three independent experiments $(p<0.05)$. Luciferase activity was calculated by normalizing firefly luciferase activity to Renilla activity. For fold changes in TCF/LEF luciferase activity relative to DMSO, see Supplementary data.

$279 \times 361 \mathrm{~mm}(300 \times 300 \mathrm{DPI})$ 
A

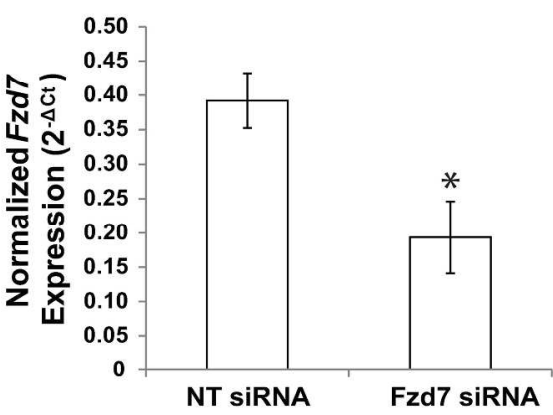

B

C
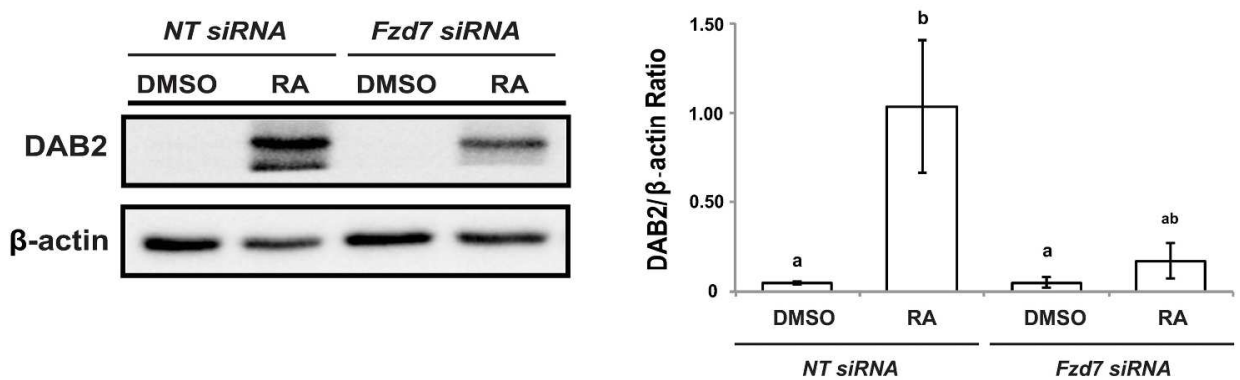

D

E
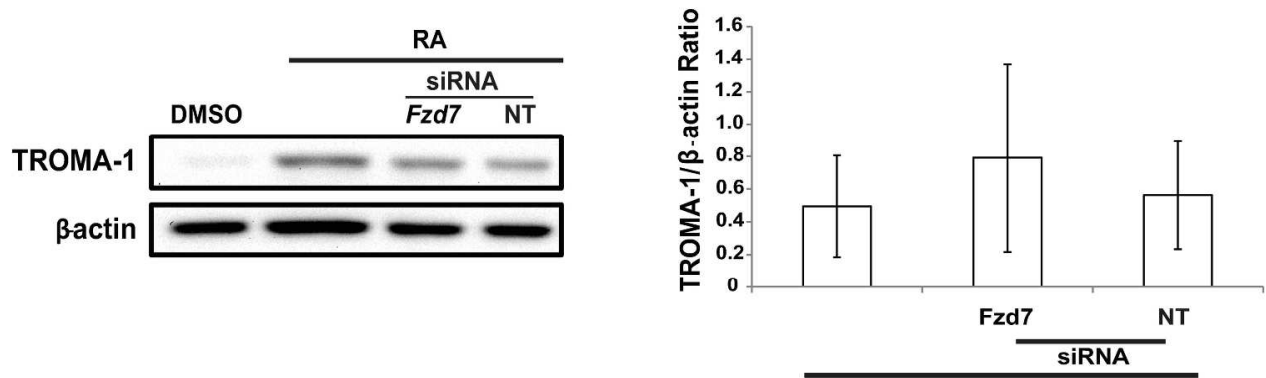

RA

Fig. 4. siRNA knockdown of Fzd7 had no apparent effect on PrE formation. F9 cells were transfected with Fzd7 siRNA or non-targeting (NT) control siRNA and treated with RA for 3 days. (A) qRT-PCR analysis indicated that $F z d 7$ mRNA expression was significantly reduced relative to levels in non-targeting control siRNA-transfected cells. (B) Immunoblot analysis showing reduced DAB2 levels in Fzd7 siRNA-transfected cells when compared to non-targeting control siRNA-transfected cells after treatment with RA. (C) Densitometric analysis confirmed that DAB2 levels were reduced in Fzd7 siRNA-transfected cells when compared to non-targeting control siRNA-transfected cells after treatment with RA. (D) Immunoblot results showed TROMA-1 levels in Fzd7 siRNA-transfected cells were approximately equal to those in the nontargeting control siRNA-transfected cells after treatment with RA, and cells treated with RA alone. (E)

Densitometric analysis confirmed that TROMA-1 levels were not significantly different in RA-treated cells transfected with Fzd7 siRNA compared to non-targeting control-transfected cells treated with RA. Antibodies against $\beta$-actin were used to ensure equal loading in all lanes. Densitometric data was collected by comparing the TROMA-1/ $\beta$-actin ratio in each sample. Data represent the mean and standard error of three 
independent experiments $(p<0.05)$.

$238 \times 293 \mathrm{~mm}(300 \times 300 \mathrm{DPI})$ 
A

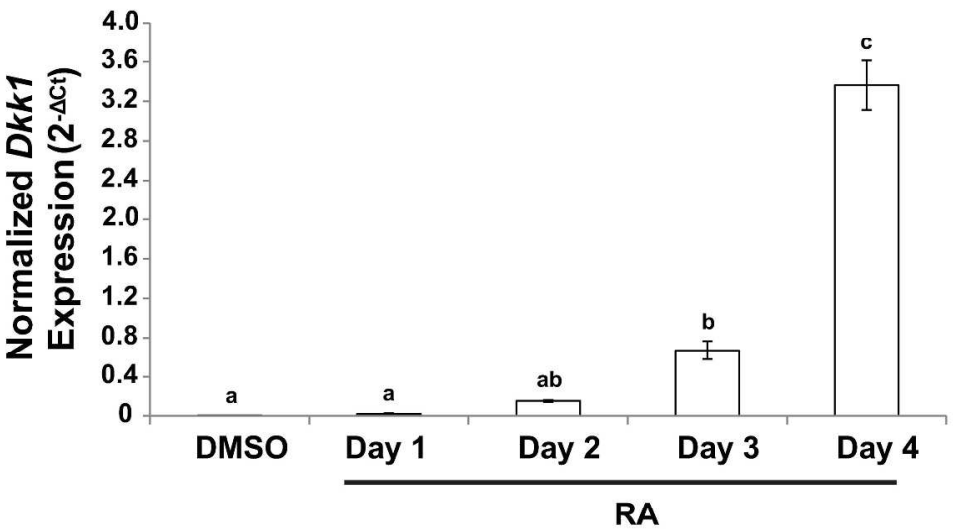

B

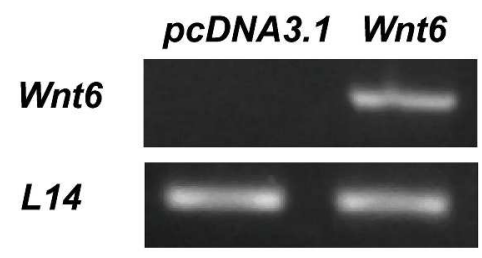

C

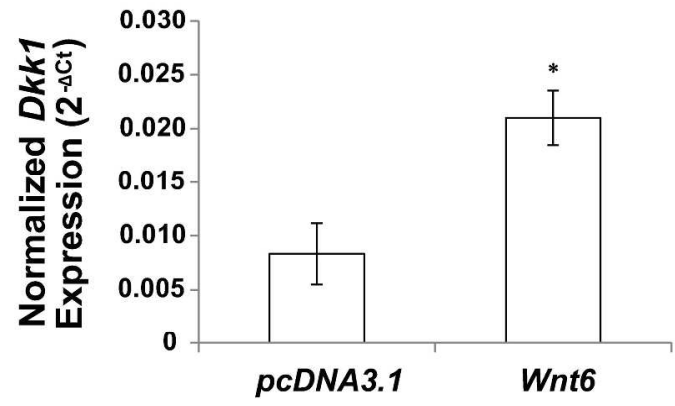

Fig. 5. Dkk1 expression following RA treatment or Wnt6 overexpression. (A) qRT-PCR analysis showed that the expression of $D k k 1$ significantly increased at 3 and 4 days after RA treatment compared to DMSOtreated cells or cells treated with RA for 1 day. Expression of $D k k 1$ at day 2 of RA treatment was not significantly different compared to DMSO-treated cells or cells treated with RA for 1 or 3 days, but was significantly different from $D k k 1$ expression at 4 days post-RA treatment. Analysis also indicated that compared to DMSO-treated cells, Dkk1 levels were increased by 119 and 611 -fold at 3 and 4 days post-RA treatment, respectively (Supplementary data). Expression of $D k k 1$ mRNA was normalized to the expression of $L 14$ mRNA. (B) End point PCR revealed the presence of a Wnt6 amplicon in cells transfected with pcDNA3.1-Wnt6, but not in those transfected with the empty vector control. (C) Total RNA was also isolated from F9 cells transfected with pcDNA3.1-Wnt6 and qRT-PCR analysis indicated that Dkk1 mRNA expression increased by 2.92-fold in Wnt6-transfected cells compared to the empty vector control. Data represent the mean and standard error of three independent experiments. Letters and $*$ denote significance $(p<0.05)$. Fold changes in Dkk1 mRNA expression relative to DMSO or empty vector treatments are presented in the 
Supplementary data.

$247 \times 365 \mathrm{~mm}(300 \times 300 \mathrm{DPI})$ 
A

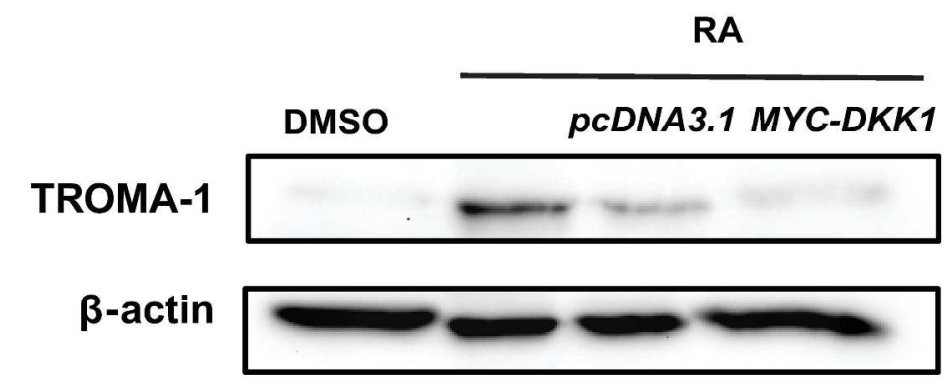

B

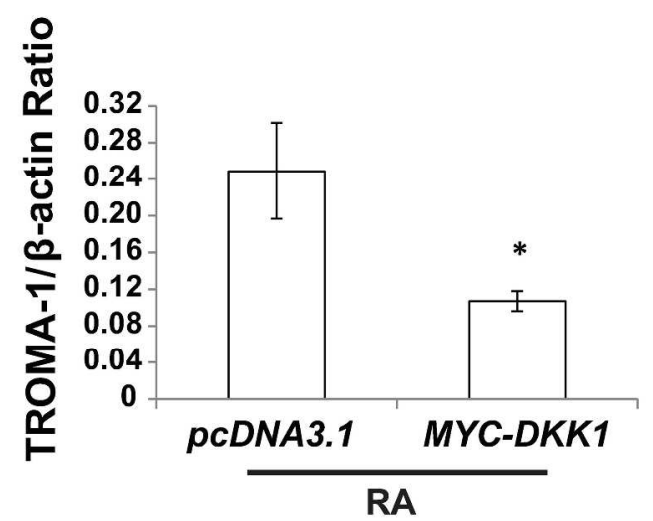

C

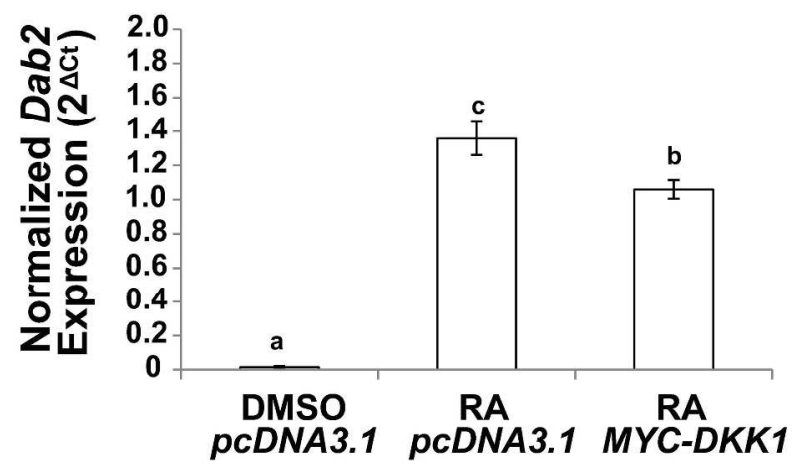

Fig. 6. F9 cells transfected with Myc-DKK1 show decreased expression of PrE markers following RA treatment. $\mathrm{F} 9$ cells were transfected with $p C D N A 3.1$ or Myc-DKK1 and treated with DMSO or RA, or treated with DMSO or RA alone. (A) Immunoblot analysis showed a TROMA-1 signal in cells transfected with pCDNA3.1 and treated with RA and cells treated with RA alone. This signal was also present in cells transfected with $M y c-D K K 1$ and treated with RA, however, its intensity was reduced in comparison to the positive controls (RA and $p c D N A 3.1+\mathrm{RA}$ ). $\beta$-actin served as a loading control. (B) Densitometric analysis confirmed that there was a significant reduction in TROMA-1 levels in cells transfected with Myc-DKK1 and treated with RA, compared to cells transfected with empty vector and treated with RA. (C) Total RNA was collected from F9 cells transfected with $M y c-D K K 1$ and treated with RA or transfected with empty vector and treated with DMSO or RA, and qRT-PCR analysis was performed using Dab2 primers. Results showed that $D a b 2$ expression was significantly reduced (by approximately 23-fold; Supplementary data) in Myc-DKK1 transfected, RA-treated cells compared to empty vector transfected, RA-treated cells. Data represent the mean and standard error of three independent experiments. * denotes significance $(p<0.05)$. 
$217 \times 353 \mathrm{~mm}(300 \times 300 \mathrm{DPI})$ 
A

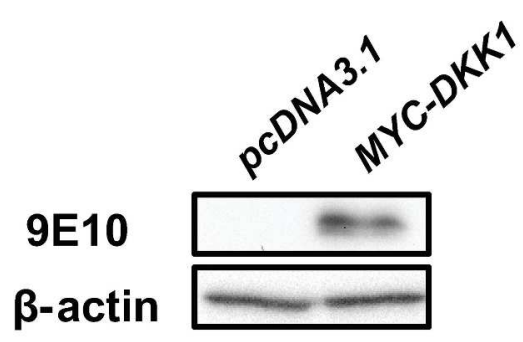

B

$\frac{\text { PcDNA3.1-CM }}{\text { DMSO RA }} \frac{\text { MYC-DKK1-CM }}{\text { RA }}$

TROMA-1

(

\section{$\beta$-a ctin}

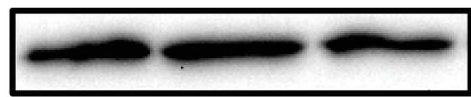

C

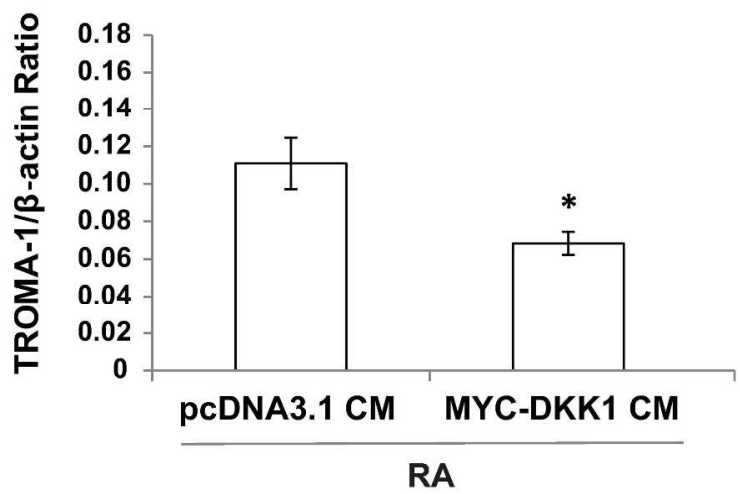

Fig.7. MYC-DKK1-conditioned media attenuated RA-induced PrE differentiation. (A) HEK-293 cells were transfected with pcDNA3.1 or Myc-DKK1 and immunoblot analysis of lysates with the $9 \mathrm{E} 10$ antibody showed the ecoptic DKK1 protein present in cells transfected with Myc-DKK1, but not pcDNA3.1. (B) F9 cells cotreated with the concentrated CM from HEK-293 cells and either DMSO or RA were collected for immunoblot analysis. A representative blot showing reduced TROMA-1 levels in cells treated with the MYC-DKK1-CM and

RA compared to pcDNA3.1-CM and RA. (C) Densitometry analysis confirmed that there was a significant decrease in TROMA-1 signal (0.39-fold) in cells co-treated with MYC-DKK1-CM and RA, compared to cells cotreated with pCDNA3.1-CM and RA. Data in (B) and (C) represent three independent experiments.* denotes significance $(p<0.05)$. 